\title{
Optimal Interconnection Circuits for VLSI
}

\author{
H. B. BAKOGLU, STUDENT MEMBER, IEEE, AND JAMES D. MEINDL, \\ FELLOW, IEEE
}

\begin{abstract}
The propagation delay of interconnection lines is a major factor in determining the performance of VLSI circuits because the $R C$ time delay of these lines increases rapidly as chip size is increased and cross-sectional interconnection dimensions are reduced. In this paper, a model for interconnection time delay is developed that includes the effects of scaling transistor, interconnection, and chip dimensions. The delays of aluminum, $\mathrm{WSi}_{2}$, and polysilicon lines are compared, and propagation delays in future VLSI circuits are projected. Properly scaled multilevel conductors, repeaters, cascaded drivers, and cascaded driver/ repeater combinations are investigated as potential methods for reducing propagation delay. The model yields optimal cross-sectional interconnection dimensions and driver/repeater configurations that can lower propagation delays by more than an order of magnitude in MOSFET circuits.
\end{abstract}

\section{INTRODUCTION}

$\mathrm{E}$ FFICIENT packaging and interconnection of silicon chips are significant challenges in high-speed digital-system design. With the evolution of computers, packaging has become the most critical aspect of the central processor in a fast mainframe computer and, as a result, sophisticated packaging technologies are required to achieve high performance [1]. Once considered to be a routine task, interconnecting the transistors of a VLSI circuit is also becoming a major concern because of the long design time required to lay out the interconnections and the interconnection propagation delay that limits overall chip performance. These problems become more severe as the minimum feature size is scaled down to the submicrometer level and chip size is increased potentially to wafer dimensions. In this paper, an $R C$ model is introduced that incorporates the resistance and capacitance of the interconnection, load capacitance, and resistance of the transistor that drives the line. This model is then used to calculate propagation delay and develop strategies to minimize it and also to predict future trends.

\section{SCALING OF INTERCONNECTION Dimensions}

In ideal scaling of MOS transistors, all linear dimensions and voltages are scaled by $1 / S$ and all doping levels are scaled by $S$, where $S$ is the scaling factor $(S>1)$. As a result, gate delay decreases by $1 / S$, power density remains the same, and the power-delay product per device is reduced by $1 / S^{3}[2],[3]$.

Manuscript received July 2, 1984; revised September 12,1984. H. B. Bakoglu is supported by an IBM Fellowship. This work was supported by DARPA under Contract MDA903-80-C-0432. Some of the earlier results of this work were presented at the IEEE International SolidState Circuits Conference, San Francisco, CA, February 1984.

The authors are with the Integrated Circuits Laboratory, Stanford University, Stanford, CA 94305.

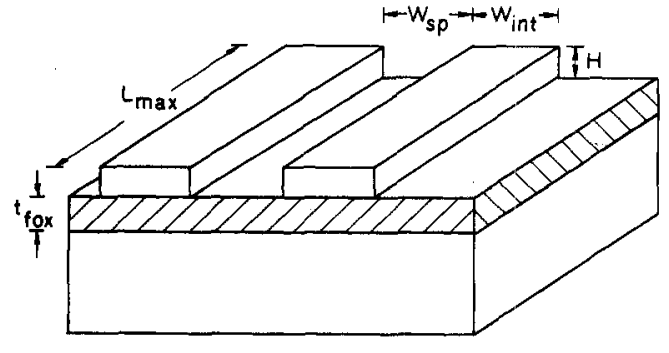

Fig. 1. Interconnection dimensions.

As device dimensions are miniaturized, the interconnection dimensions (Fig. 1) must also be reduced to take full advantage of the scaling process.

A straightforward approach to satisfying the processing and layout constraints is to scale all cross-sectional interconnection dimensions ( $W_{\text {int }}, W_{s p}, H, t_{\text {fox }}$ ) by the same factor as used for transistors (ideal scaling). The devices and interconnections would then require the same relative accuracy from lithography, pattern-etching, and material-deposition technologies, and the aspect ratios of interconnections and steps will not change as sizes are reduced. The effects of ideal scaling on local and longdistance interconnections are listed in Tables I and II [4]. Although gate delay decreases by $1 / S$ in ideal scaling, it can be seen that the response time of local interconnections remains the same. Even more troublesome, the delay time of longdistance interconnections (such as those extending from corner to corner on a die) increases by $S^{2} S_{c}^{2}$, where $S_{c}$ is the scaling factor for the chip size. The chip scaling factor is included to account for the increase in die size from one generation of integrated circuits to the next.

Deviation from ideal scaling can be advantageous. Scaling interconnections and field-oxide thickness by factors smaller than $S$ will lower $R_{\text {int }}$ and $C_{\text {int }}$. Two alternative approaches to minimizing propagation delay are also presented in Tables I and II. In "quasi-ideal" scaling of local interconnections, the horizontal dimensions are scaled by $1 / S$ (like transistors) to improve overall packing density by a factor $S$. On the other hand, the vertical dimensions are reduced only by $1 / \sqrt{S}$ to maintain a small $R C$ time constant and, as a result, delay decreases by $1 / \sqrt{S}$. In "constant- $R$ " scaling, all cross-sectional dimensions are reduced only by $1 / \sqrt{S}$ and, consequently, propagation delay is lowered by $1 / S$. Long-distance interconnections are more difficult because of the additional burden introduced by the increasing chip size. In "constant-dimension" scaling of long-distance interconnections, all cross-sectional dimensions are held constant, and propagation delay rises by $S_{c}^{2}$ 
TABLE I

SCALING OF LOCAL INTERCONNECTIONS

\begin{tabular}{|c|c|c|c|c|}
\hline Parameter & $\begin{array}{l}\text { Ideal } \\
\text { Scaling }\end{array}$ & $\begin{array}{l}\text { Quasi-Ideal } \\
\text { Scaing }\end{array}$ & $\begin{array}{l}\text { Constant-l } \\
\text { Scaling }\end{array}$ & $\begin{array}{c}\text { Generalized } \\
\text { Sculing } \\
\end{array}$ \\
\hline I'hicknomit (/I) & $1 / 5$ & $1 / \sqrt{5}$ & $1 / \sqrt{5}$ & $1 / s_{n}$ \\
\hline$\left.M M_{1}\right)\left(H_{i n t}\right)$ & $1 / 5$ & $1 / 5$ & $1 / \sqrt{5}$ & $1 / t_{\text {i.ut }}$ \\
\hline Söpritrition $\left(111_{i p}\right)$ & $1 / 5$ & $1 / 5$ & $1 / \sqrt{5}$ & $1 / S_{u p}$ \\
\hline Fiedd-oxint thickness(l,os) & $1 / 5$ & $1 / \sqrt{6}$ & $1 / \sqrt{5}$ & $1 / s_{\text {gos }}$ \\
\hline lethgt $]_{1}\left(I_{10 \mathrm{oc}}\right)$ & $1 / .5$ & $1 / 5$ & $\approx 1 / 9$ & $1 / 5$ \\
\hline Rouletenter $\left(R_{i n 1}\right)$ & 5 & $\sqrt{5}$ & 1 & $S_{f n t} S S_{m} / s$ \\
\hline 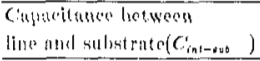 & $1 / S$ & $1 / 5^{3 / 2}$ & $\approx 1 / \mathrm{s}$ & $S_{S a s} / S S_{i u t}$ \\
\hline 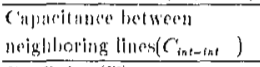 & $1 / 5$ & $1 / \sqrt{S}$ & $\approx 1 / S$ & $S_{* p} / 5 S_{H}$ \\
\hline$P C D M a y(T)$ & 1 & $\approx 1 / \sqrt{5}$ & $\approx 1 / 5$ & $\approx S_{\text {int }} S_{A} / S^{0}$ \\
\hline Voltage drop (IR) & 1 & $1 / \sqrt{s}$ & $1 / 5$ & $s_{\text {int }} s_{H 1} / S^{2}$ \\
\hline Curron! densily $(J)$ & $s$ & $\sqrt{5}$ & 1 & $S_{\text {int }}, S_{n} / S$ \\
\hline
\end{tabular}

TABLE II

SCALING OF LONG-DISTANCE INTERCONNECTIONS

\begin{tabular}{|c|c|c|c|c|}
\hline Purameter & $\begin{array}{l}\text { Ideal } \\
\text { Scaling }\end{array}$ & $\begin{array}{l}\text { Constant } \\
\text { Dimenston }\end{array}$ & $\begin{array}{l}\text { Constant } \\
\text { Delay }\end{array}$ & $\begin{array}{c}\text { Goneralizet } \\
\text { scaling }\end{array}$ \\
\hline Thipkuess (II) & $1 / S$ & 1 & $s$ & $1 / \varphi_{14}$ \\
\hline Width $\left(11_{i n t}\right)$ & $1 / s$ & 1 & $S_{e}$ & $1 / S_{\text {int }}$ \\
\hline Separation $\left(1 k_{p p}\right)$ & $1 / 5$ & 1 & 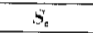 & $1 / s_{s}$ \\
\hline 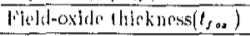 & $1 / 5$ & 1 & $S_{s}$ & $1 / s^{\prime 6 x}$ \\
\hline$L_{\text {Inllgh }}\left(l_{\text {mius }}\right)$ & 5 & $S_{s}$ & $S_{8}$ & $S_{c}$ \\
\hline Remistunen $\left(R_{i n 1}\right)$ & $S^{3} S_{8}$ & $s_{c}$ & $1 / S_{s}$ & $S_{i n t} S_{t s} S_{c}$ \\
\hline Capactuner $\left(C_{\text {int }}\right)$ & $S_{e}$ & $S_{i}$ & s. & $\approx s_{0}$ \\
\hline$R($ Dellay $(T)$ & $S^{2} S_{a}{ }^{2}$ & $S_{e}^{2}$ & 1 & $S_{i n t} S_{\|} S_{a}{ }^{2}$ \\
\hline
\end{tabular}

because of the growth in chip size. In "constant-delay" scali ig, the cross-sectional interconnection dimensions are increa:ed such that the improvement in the $R C$ delay per unit lenfrth cancels the effect of increasing chip size and total delay remains constant.

As the ratios $H / W_{\text {int }}$ and $t_{f \circ x} / W_{\text {int }}$ get larger, the two-dim sional fringing fields and capacitances between neighboring lines become important and, after a certain point, larger aspict ratios yield no additional advantage. As a result, the capcacitance per unit length of the wires in a multilevel interconns:tional scheme approaches a lower limit of $2 \mathrm{pF} / \mathrm{cm}$ with $\mathrm{SiO}_{2}$ as the dielectric material; an ultimate limit of $1 \mathrm{pF} / \mathrm{cm}$ is $\mathrm{plo}-$ jected with improved dielectric materials [5]. To obtain :ccurate results, two-dimensional fringing fields and the contribution of the neighboring lines must be included in determining the capacitance of the interconnections; neglecting these efferts can introduce substantial error. Expressions that are used to calculate the capacitances in the following derivations have liss than 5-percent error over a wide range of interconnection parameters; a set of design rules and interconnection dimensio ns is assumed, and a capacitance of $3 \mathrm{pF} / \mathrm{cm}$ is achieved independent of minimum feature size with ideal scaling. This result is in agreement with detailed analytical and computer-aided mode is developed to determine the capacitance of VLSI interconnections [5]-[14]. In the following calculations, conductivity is assumed to remain constant, and this is a valid assumption un i1 the mean-free path of the carriers becomes comparable to interconnection thickness and width; however, this does not become a problem until the interconnection width is scaled dow $n$

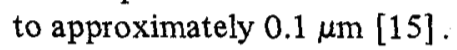

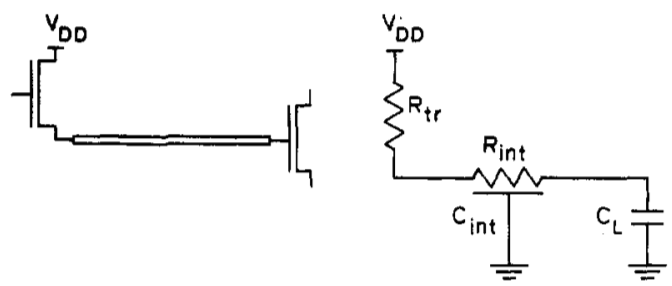

Fig. 2. Interconnection-delay model.

\section{MODEL FOR INTERCONNECTION Propagation Delay}

Interconnection delay is modeled as illustrated in Fig. 2. Under step-voltage excitation, the times $(T)$ required for the output voltage of distributed and lumped $R C$ networks to rise from 0 to 90 percent of their final values are $1.0 R C$ and $2.3 R C$, respectively [16]. Accordingly, a very good approximation for delay is obtained by combining the resistive and capacitive terms and weighting them by the appropriate factors as described earlier

$$
\begin{aligned}
& T=1.0 R_{\mathrm{int}} C_{\mathrm{int}}+2.3\left(R_{t r} C_{\mathrm{int}}+R_{t r} C_{L}+R_{\mathrm{int}} C_{L}\right) \\
& T \approx\left(2.3 R_{t r}+R_{\mathrm{int}}\right) C_{\mathrm{int}} .
\end{aligned}
$$

This approximation is in agreement with Sakurai's expression [17] reported to have less than 4-percent error over the entire range of parameters. The on-resistance of a MOS transistor is

$$
R_{t r} \approx \frac{L / W}{\mu C_{g \circ \mathrm{x}} V_{D D}}
$$

which remains constant with ideal scaling. Interconnection resistance is

$$
R_{\text {int }}=\rho \frac{L_{\max }}{W_{\text {int }} H}
$$

and, as shown in Table II, it increases as $S^{2} S_{c}$ when ideal scaling is applied. The capacitance of the center one of three adjacent lines above a ground plane is expressed [7] as

$$
\begin{aligned}
\frac{C_{\mathrm{int}}}{\epsilon_{\mathrm{ox}} L_{\max }}= & 1.15\left(\frac{W_{\text {int }}}{t_{\text {fox }}}\right)+2.80\left(\frac{H}{t_{\text {fox }}}\right)^{0.222} \\
& +\left(0.06\left(\frac{W_{\mathrm{int}}}{t_{f \circ \mathrm{x}}}\right)+1.66\left(\frac{H}{t_{f \circ \mathrm{x}}}\right)\right. \\
& \left.-0.14\left(\frac{H}{t_{f \circ \mathrm{x}}}\right)^{0.222}\right)\left(\frac{t_{f \circ \mathrm{x}}}{W_{s p}}\right)^{1.34}
\end{aligned}
$$

with an error of less than 10 percent over a wide range of $H /$ $t_{\text {fox }}, W_{\text {int }} / t_{\text {fox }}$ and $W_{s p} / t_{f o x}$ ratios.

Fig. 3 plots the propagation delay for various chip dimensions as a function of minimum feature size and for two driver resistances $(1$ and $10 \mathrm{k} \Omega$ ). It can be seen that the delay is dominated by $R_{\mathrm{int}} C_{\mathrm{int}}$ as dimensions are scaled aggressively. With the existing aspect ratios and design rules, the capacitive term is determined by the capacitance between the interconnection and substrate $\left(\approx \epsilon_{\mathrm{ox}} L_{\max } W_{\text {int }} / t_{\text {fox }}\right)$. In modest VLSI circuits and for aluminum lines, the resistive term is dominated by $R_{t r}$ and, in larger VLSI circuits and for polysilicon lines, it is con- 


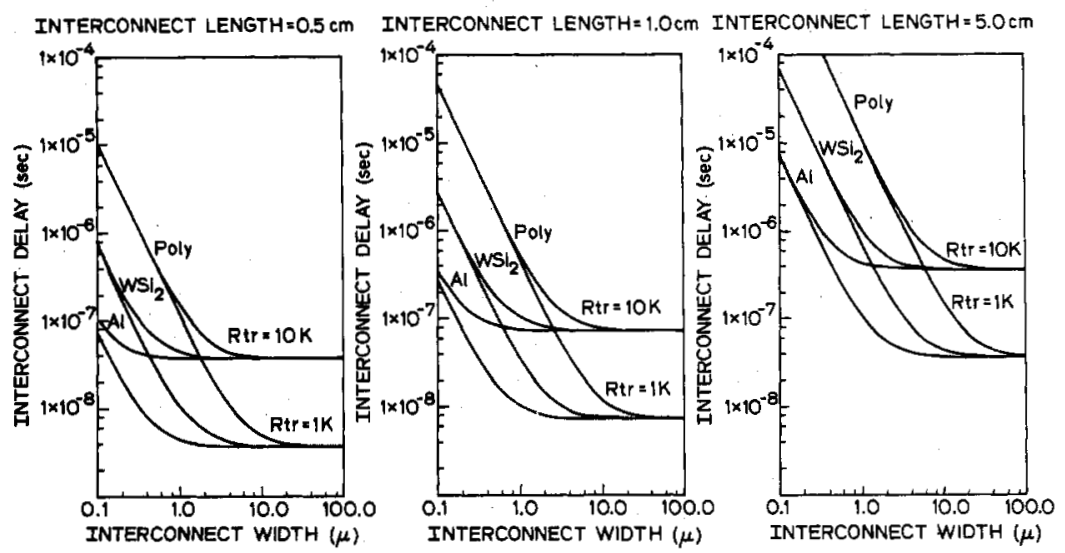

Fig. 3. Interconnection delay as a function of width for three materials (Al, $W \mathrm{Wi}_{2}$, polysilicon), two driver resistances $1 \mathrm{k} \Omega(W / L=10)$ and $10 \mathrm{k} \Omega(W / L=1)$, and three lengths $(0.5,1.0,5.0 \mathrm{~cm})$. It is assumed that $W_{s p}=W_{\text {int }}, H=W_{\text {int }} / 3, t_{\text {fox }}=W_{\text {int }} / 5$, and ideal scaling is applied. Also, $\rho_{\mathrm{Al}}=3 \mu \Omega \cdot \mathrm{cm}, \rho_{\mathrm{WSi}_{2}}=30 \mu \Omega \cdot \mathrm{cm}$, and $\rho_{\text {Poly }}=500 \mu \Omega$. $\mathrm{cm}$.

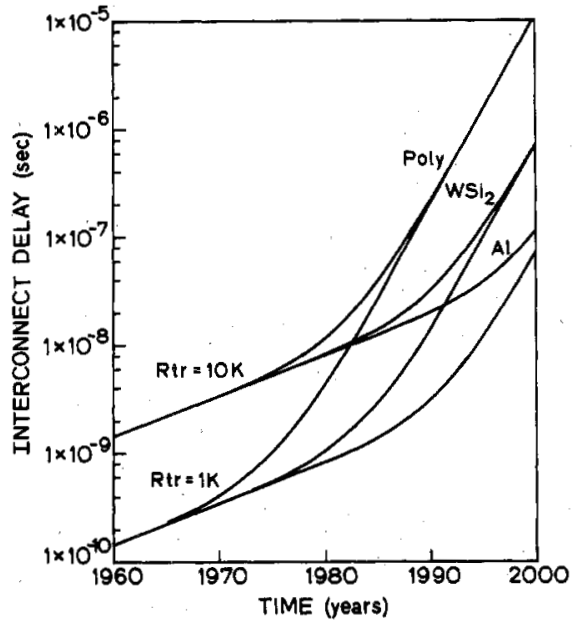

Fig. 4. Projected long-distance propagation delays of future integrated circuits with single-layer metal interconnections. Equations (7) and (8) are used to determine $\lambda$ and $L_{\max }$. It is assumed that $W_{\text {int }}=$ $\frac{3}{2} \lambda, W_{s p}=\frac{3}{2} \lambda, H=\frac{1}{4} \lambda, t_{f o x}=\frac{1}{6} \lambda$, and ideal scaling is applied.

trolled by $R_{\text {int }}$. The optimal interconnection dimensions for a given length can be obtained from the corner point of the curves where $R_{\text {int }} \approx 2.3 R_{t r}$. The optimal cross-sectional dimensions, therefore, are

$$
W_{\text {int }} H=\rho \frac{L_{\max }}{2.3 R_{t r}} .
$$

The projected long-distance interconnection propagation delays of future integrated circuits with single-layer metal are plotted in Fig. 4. Here, the minimum feature size is assumed to drop by a factor of 2 every six years and chip dimension is assumed to double every eight years as

$$
\begin{aligned}
\lambda & =2.0 \times 2^{-(t-1983) / 6} \mu \mathrm{m} \\
L_{\max } & =0.35 \times 2^{(t-1983) / 8} \mathrm{~cm} .
\end{aligned}
$$

It can be seen that, in the early 1970 s, all interconnections were pure capacitive loads, and time delay was determined by the size of the driver and the length of the interconnection. As the chip dimension increases and the minimum feature size decreases, $R_{\text {int }}$ becomes important and propagation delay rises rapidly. Today, aluminum lines still can be approximated as capacitive loads, whereas polysilicon lines have exhibited an $R C$ behavior for some time. If the conventional methods for interconnecting the transistors are not improved, longer interconnection lengths and smaller widths and thicknesses will result in unacceptably long propagation delays. Since in the past the channel of the transistor dominated the resistive component of delay and the interconnections were virtually perfect conductors with respect to the channel and the parasitic capacitances of all components (gate, diffusion, polysilicon, aluminum) have been significant, the major challenges were to minimize these capacitances and to drive large capacitive loads. With smaller feature sizes and greater chip dimensions, the parasitic resistances of interconnections are becoming comparable to channel resistance and, as a result, the design challenges are to reduce parasitic resistances and capacitances and to drive large $R C$ rather than large capacitive loads.

\section{Methods for Improving Propagation Delay}

Two approaches to shortening interconnection delay are investigated. The first is to reduce interconnection resistance by using only aluminum lines for long-distance communication and by forming multilayers of interconnections with thicker and wider lines in the upper levels. The second is to improve the driver circuit through cascaded drivers that increase in size until the last device is large enough to drive the line and/or by using repeaters that divide the interconnection into smaller subsections [18].

\section{A. Multilayer Interconnections}

It is not possible to interconnect the transistors with only one level of aluminum without polysilicon or diffusion crossovers; moreover, even aluminum lines will introduce excessive delay when chip dimension and minimum feature size are scaled. Multilayers of interconnections are partial solutions to both 


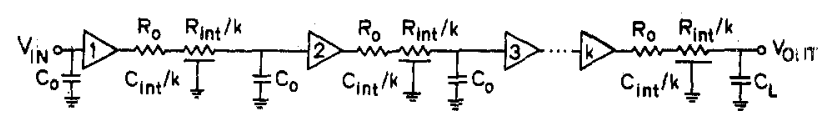

(a)

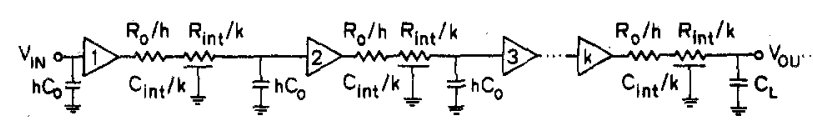

(b)

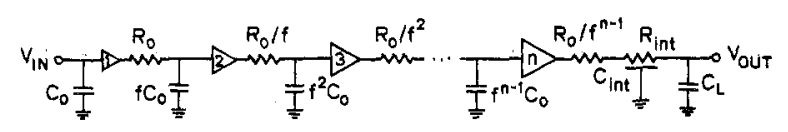

(c)
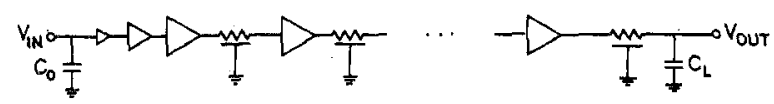

(d)

Fig. 5. Methods for driving interconnections. (a) Minimum size repeaters. (b) Optimal repeaters. (c) Cascaded drivers. (d) Optimal. repeaters with a cascaded first stage.

problems. Layers of aluminum in the $x$-and $y$-directions interconnected through vias between the levels enable long-distarice communication without the need for polysilicon or diffusion crossovers. Based on (6), the cross-sectional dimensions of the upper layers can also be optimized to reduce propagation dela//s; the local interconnections can use the scaled down first-level metal, and the long-distance interconnections can use the uprer levels with wider and thicker lines that yield shorter proparation delays. Because much of the chip area is occupied by interconnections, multilayers of metal can also reduce chip size and further improve the time delay because the average intr. $r$ connection length is inversely proportional to the number of levels [5], [19].

\section{B. Repeaters}

When the resistance of the interconnection is comparable :o or larger than the on-resistance of the driver, propagatic $n$ delay increases as the square of the interconnection length $\mathrm{be}$ cause both capacitance and resistance increase linearly will length. The use of repeaters makes time delay linear. wilh length by dividing the interconnection into smaller subsectior s. Based on the model developed in the preceeding section, tle propagation delay of an interconnection with $k$ minimum-size inverters as repreaters (Fig. 5(a)) can be expressed as

$$
T=k\left(2.3 R_{0}+\frac{R_{\text {int }}}{k}\right)\left(\frac{C_{\text {int }}}{k}+C_{0}\right)
$$

where $C_{0}$ and $R_{0}$ are the input capacitance and output resi:tance of the minimum size inverter. By setting $d T / d k=0$

$$
\frac{R_{\mathrm{int}} C_{\mathrm{int}}}{k^{2}}=2.3 R_{0} C_{0}
$$

To achieve the shortest total delay, therefore, the delay of th: segments connected by the repeaters should be equal to tha of a repeater. The number of repeaters is

$$
k=\sqrt{\frac{R_{\text {int }} C_{\text {int }}}{2.3 R_{0} C_{0}}} .
$$

Total delay is then expressed as

$$
T=\left(\sqrt{2.3 R_{0} C_{\text {int }}}+\sqrt{R_{\text {int }} C_{0}}\right)^{2} .
$$

For long-distance interconnections, $C_{\text {int }}$ is on the order of picofarads and $C_{0}$ is on the order of femptofarads, and $R_{\text {int }}$ and $R_{t r}$ have values around kilohms; therefore, $R_{0} C_{\text {int }} \gg>$ $R_{\text {int }} C_{0}$, and the delay expression can be further simplified to

$$
T \approx 2.3 R_{0} C_{\text {int }} \text {. }
$$

As a result, repeaters can effectively "transform" the interconnection into a capacitive load.

Propagation time can be further improved by increasing the size of the repeaters because the current-drive capability of the repeaters is directly proportional to the $W / L$ ratios of its devices. When the $W / L$ ratios of the transistors are increased by a factor $h$, the output resistance and input capacitance become $R_{0} / h$ and $h C_{0}$, respectively (Fig. $5(\mathrm{~b})$ ), and the delay expression then takes the form of

$$
T=k\left(2.3 \frac{R_{0}}{h}\left(\frac{C_{\text {int }}}{k}+h C_{0}\right)+\frac{R_{\text {int }}}{k}\left(\frac{C_{\text {int }}}{k}+2.3 h C_{0}\right)\right) .
$$

By setting $d T / d k$ and $d T / d h$ to zero, optimal values for $k$ and $h$ are obtained as

$$
\begin{aligned}
& k=\sqrt{\frac{R_{\mathrm{int}} C_{\mathrm{int}}}{2.3 R_{0} C_{0}}} \\
& h=\sqrt{\frac{R_{0} C_{\mathrm{int}}}{R_{\mathrm{int}} C_{0}}}
\end{aligned}
$$

and resulting delay expression becomes

$$
T=7.6 \sqrt{R_{0} R_{\mathrm{int}} C_{0} C_{\mathrm{int}}}
$$

which is shorter than the delay achieved by minimum-size repeaters because $R_{0} C_{\text {int }}>>R_{\text {int }} C_{0}$.

\section{Cascaded Drivers}

Just as repeaters are good in driving large $R C$ loads, cascaded drivers are good in driving large capacitive loads. Instead of a single minimum-size driver, a chain of drivers can be used that increase in size until the last device is large enough to drive the load [20]. This is necessary because, if the load is driven by a large transistor which, in turn, is driven by a small device, the turn-on time of the large transistor dominates the delay term. The optimal delay is obtained with a sequence of drivers that increase gradually in size. When applied to interconnections (Fig. 5(c)), this method optimizes the sum of the delay caused by charging the input capacitances of the cascaded drivers and the interconnection propagation delay. Total delay is then expressed as

$$
T=2.3(n-1) f R_{0} C_{0}+\left(\frac{2.3 R_{0}}{f^{n-1}}+R_{\text {int }}\right) C_{\text {int }}
$$


and, by setting $d T / d n$ and $d T / d f$ to zero

$$
\begin{aligned}
& f=e \\
& n=\ln \left(\frac{C_{\text {int }}}{C_{0}}\right) \\
& T=2.3 e R_{0} C_{0} \ln \left(\frac{C_{\text {int }}}{C_{0}}\right)+R_{\text {int }} C_{\text {int }}
\end{aligned}
$$

where $e$ is the base of the nautral logarithm. Note that cascaded drivers minimize the $R_{t r} C_{\text {int }}$ term in (2), but the $R_{\text {int }} C_{\text {int }}$ term remains unchanged. As a result, this method is very useful when $R_{\text {int }}$ is small and $C_{\text {int }}$ and $R_{0}$ are dominant; however, it is not adequate when $R_{\text {int }}$ is comparable to or larger than $R_{0}$. In addition, because the total time delay increases slowly. with increased $f$, in practice a size ratio $f$ larger than $e$ is used to save chip area with little increase in propagation time.

As illustrated in Fig. 5(d), the first stage of the optimal-size repeaters must be a cascaded driver to lower the input capacitance of the structure and to optimize the total propagation delay when it is driven by a minimum-size transistor.

Figs. 6 and 7 compare the different driving schemes. It can be seen that repeaters eliminate the $R_{\text {int }}$ term and that delay increases linearly with $L_{\max }$ because $C_{\text {int }}$ is linearly proportional to length. Cascaded drivers are preferred when $L_{\max }$ is short because interconnection resistance is low for small $L_{\max }$; however, for longer $L_{\max }$, delay increases rapidly because cascaded drivers do not improve the $R_{\text {int }} C_{\text {int }}$ term. Optimal size repeaters with a cascaded first stage combine the advantages of the two and obtain the shortest delay under all conditions. The ultimate lower limit is the propagation delay of a lossless transmission line; here the transmission speed is

$$
v=\frac{c}{\sqrt{\epsilon_{r}}}
$$

where $c$ is the speed of light in vacuum and $\epsilon_{r}$ is the dielectric constant of the medium in which the line is buried.

\section{Additional Considerations}

All of the preceding methods introduce area penalties to improve performance, and the models reported here do not include the possible increase in the average interconnection length and resulting complications. In addition to interconnection delay, the designer must consider the total area required to implement the circuit and the yield variation caused by process complexity introduced by some of the described methods (multilevel interconnections). After these approaches have been modified to meet the constraints of a specific IC fabrication line, they can then be included in the computer-aided design tools to optimize propagation delays automatically.

Source and drain resistances in addition to the contact resistances of MOS transistors become major concerns as minimum feature size and junction depths are scaled down [13], [21], [22]. These problems can be resolved by silicidation of the source and drain areas and by new contact materials and methods [23] , [24]. These parasitic resistances can also be included in the delay model by combining them with the on-resistance of the transistor.
The interconnection resistance is larger than the value obtained in (4) because of the reduced cross-sectional area at the steps. This is dependent to the specific metal deposition system, and the model can be adjusted to include this effect by experimentally determining a factor by which $L_{\max }$ can be multiplied to obtain an effective $L_{\max }$.

As $W_{s p}$ is reduced and $H$ is increased the capacitive crosstalk between interconnections becomes significant. In addition, the inductive coupling increases because inductance rises logarithmically as the linewidth of the interconnection is scaled down, and magnetic couplings have a longer range than do capacitive couplings [6]. Because this may become very important in future VLSI circuits, the capacitive and inductive couplings between interconnections must be analyzed carefully as the linewidth is reduced and chip size increases.

\section{Effects of Interconnection Time Delay}

In the model developed in this investigation, a single transistor drives one interconnection line; however, a single transistor must often drive many signal lines. A dramatic example is the clock driver and clock lines. As chip size increases, the network of clock lines constitute a large $R C$ load that produces excessive clock skew and sets an upper limit on clock frequency. Clock frequency determines the overall performance of the chip (cycle time, access time, instructions per second) because, despite its fast switching speed, a transistor must normally wait until the next clock cycle before it can change its state. In MSI or LSI circuits, maximum clock frequency is mainly determined by the switching speed of the gates; however, in advanced VLSI circuits, clock skew caused by interconnection delay becomes the dominant delay term and limits overall chip performance. Radical changes in design methods (such as selftimed systems) have been proposed as possible solutions to this problem [25]; however, it would be difficult to address the design complexity of VLSI circuits without a clock signal that serves as a convenient sequence and time reference [26] . To enhance performance, an intermediate approach may be a highly partitioned integrated circuit with a collection of clock signals (fastest clock for the smallest partition, slowest clock for overall chip communication). These limitations and methods will become even more significant with the advent of waferscale integration.

In addition, by using the model for interconnection propagation delay, the optimal chip size for a state-of-the-art multichip packaging technology can be determined. Given the characteristic parameters of chip- and package-level interconnections, the best performance will be achieved when the interconnection delay on the chip is roughly equal to the delay of the chip-to-chip interconnections. If the chips are made larger, the system will become slower; if they are made smaller, the complexity of the package will increase with no speed improvement. Chip- and package-level delays can be set equal, assuming optimal driving schemes, to obtain

$$
7.6 \sqrt{R_{0} R_{\mathrm{int}} C_{0} C_{\mathrm{int}}}=2.3 e R_{0} C_{0} \ln \left(\frac{C_{\mathrm{INT}}}{C_{0}}\right)+R_{\mathrm{INT}} C_{\mathrm{INT}} .
$$




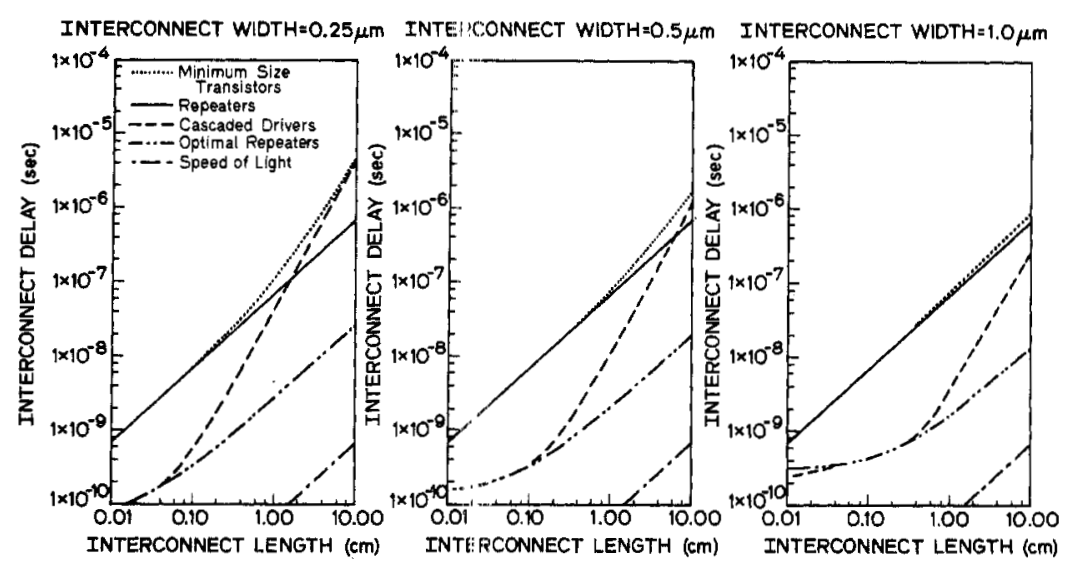

(a)

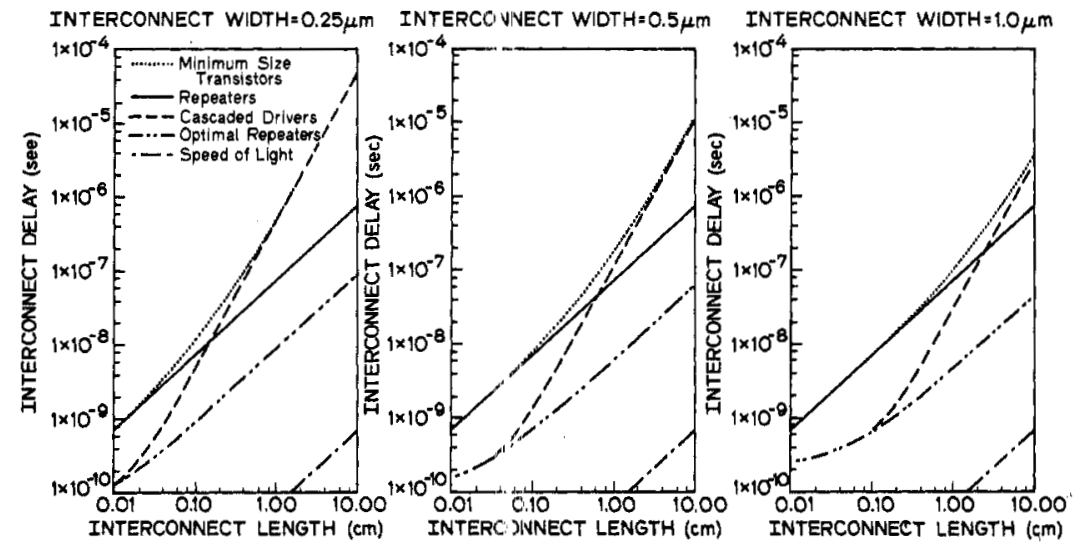

(b)

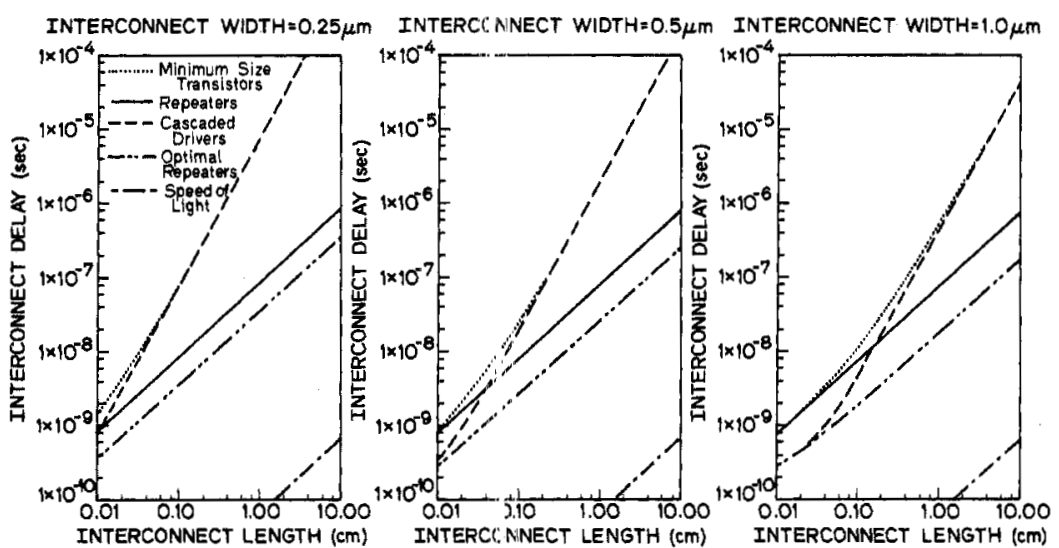

(c)

Fig. 6. Comparison of interconnuction driving methods. Propagation delay is plotted for four driving mechanisms as a function of interconnection length and for three wict ths $(0.25,0.5,1.0 \mu \mathrm{m})$. It is assumed that $W_{s p}=W_{\text {int }}, H=W_{\text {int }} / 3, t_{f i z x}=W_{\text {int }} / 5, t_{g \circ x}=W_{\text {int }} / 75$, and the minimum gate dimension is $2 W_{\mathrm{nt}} / 3$. As a result, $R_{0}=10 \mathrm{k} \Omega, C_{0}=$ $1.17 \times 10^{-15} \mathrm{~F} / \mu \mathrm{m} \times W_{\text {int }}, C_{\text {int }}=3.0 \times 10^{-12} \mathrm{~F} / \mathrm{cm} \times L_{\max }$, and $R_{\text {int }}=3.0 \times p L_{\max } / W_{\text {int }}^{2}$. (a) Aluminum. (b) $\mathrm{WSi}_{2}$. (c) Polysilicon.

Because the package level $R_{\text {INT }}$ is usually negligible

$$
\begin{aligned}
\sqrt{R_{\text {int }} C_{\text {int }}} & =0.82 \sqrt{R_{0} C_{0}} \ln \left(\frac{C_{\text {INT }}}{C_{0}}\right) \\
L_{\text {max }} & =0.82 \sqrt{\frac{R_{0} C_{0}}{r_{\text {int }} c_{\text {int }}}} \ln \left(\frac{c_{\text {INT }}}{C_{0}} L_{\mathrm{MAX}}\right)
\end{aligned}
$$

where $r_{\text {int }}, c_{\text {int }}$, and $c_{\text {INT }}$ are the resistance and capacitance per unit length, and the lower- and upper-case subscripts refer to the chip and package levels, respectively. Using $L_{\max }=\sqrt{A} / 2$, where $A$ is the area of the die or package [27], the optimal chip size is obtained as a function of the package size and characteristic interconnection parameters at the chip and package levels 


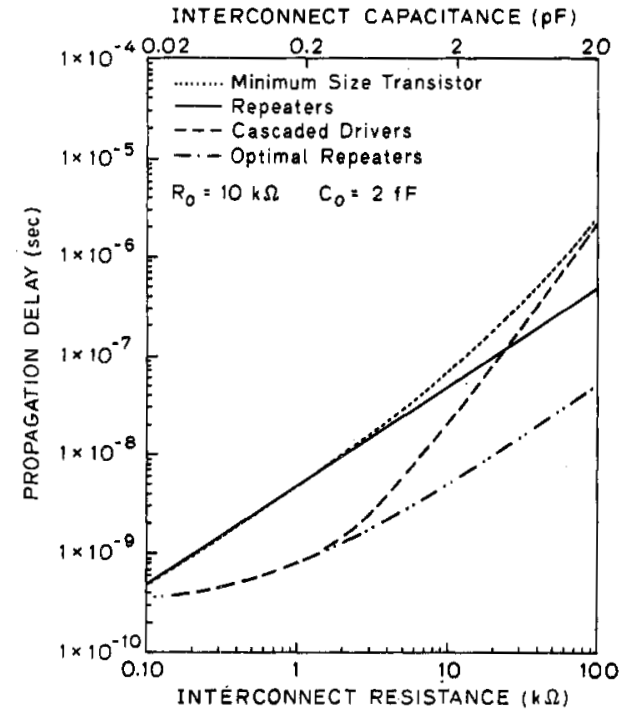

Fig. 7. Comparison of interconnection driving methods as a function of $R_{\text {int }}$ and $C_{\text {int }}$.

$$
\begin{gathered}
\sqrt{A_{\text {chip }}}=1.6 \sqrt{\frac{R_{0} C_{0}}{r_{\text {int }} c_{\text {int }}}} \ln \left(\frac{c_{I N T}}{C_{0}} \frac{\sqrt{A_{\text {package }}}}{2}\right) . \\
\text { VI. CONCLUSION }
\end{gathered}
$$

The propagation delay of interconnections is a major factor in determining the performance of VLSI circuits because the $R C$ time constant of these lines increases rapidly as chip and device dimensions are scaled. This behavior affects bipolar circuits and GaAs-based IC's even more than it does MOS circuits. The performance advantage of bipolar transistors and GaAs devices over MOS transitors is their low output impedance which is significant if the load is capacitive; however, it is less important if the transistor drives an $R C$ load with a resistance larger than the output resistance of the transistor.

Multilayers of interconnections improve propagation delay significantly and are essential for high-performance VLSI. With multilayers, all the long-distance interconnections can be formed from low-resistivity aluminum lines, and the crosssectional dimensions of the upper layers can be adjusted to yield shorter time delays. Multilayers also occupy less chip area, which reduces interconnection length and again improves propagation delay.

Repeaters eliminate the $R_{\text {int }} C_{\text {int }}$ term in the delay expression and effectively "transform" the interconnection into a capacitive load. Cascaded drivers optimize the driving of a capacitive load, but the $R_{\text {int }} C_{\text {int }}$ term remains unchanged. Optimal size repeaters with a cascaded first stage combine the advantages of the two methods and obtain the shortest delay under all condi. tions. The ultimate lower limit for the time delay is set by the propagation speed of a signal in a lossless transmission line, and this limit is approached as parasitic resistances are eliminated.

\section{ACKNOWLEDGMENT}

The encouragement of P. Losleben and R. Reynolds are gratefully acknowledged.

\section{REFERENCES}

[1] A. J. Blodgett, "Microelectronic packaging," Sci. Amer., pp. 8696 , July 1983.

[2] R. H. Dennard, F. H. Gaensslen, H. N. Yu, V. L. Rideout, E. Bassous, and A. R. LeBlanc, "Design of ion implanted MOSFET's with very small physcial dimensions," IEEE $J$. Solid-State Circuits, vol. SC-9, pp. 256-268, Oct. 1974.

[3] VLSI Laboratory, Texas Instruments Inc., "Technology and design challenges of MOS VLSI," IEEE J. Solid-State Circuits, vol. SC-17, pp. 442-448, June 1982.

[4] K. C. Saraswat and F. Mohammadi, "Effect of scaling of interconnections on the time delay of VLSI circuits," IEEE J. SolidState Circuits, vol. SC-17, pp. 275-280, Apr. 1982.

[5] P. M. Solomon, "A comparison of semiconductor devices for high speed logic," Proc. IEEE, vol. 70, pp. 489-509, May 1982.

[6] H. Yuan, Y. Lin, and S. Chiang, "Properties of interconnection on silicon, sapphire, and semi-insulating gallium arsenide substrates," IEEE Trans. Electron Devices, vol. ED-29, pp. 639644, Apr. 1982.

[7] T. Sakurai and T. Tamaru, "Simple formulas for two- and threedimensional capacitances," IEEE Trans. Electron Devices, vol. ED-30, pp. 183-185, Feb. 1983.

[8] A. E. Ruehli, "Survey of computer-aided electrical analysis of intergrated circuit interconnections," IBM J. Res. Develop., vol. 23, pp. 626-639, Nov. 1979.

[9] A. E. Ruehli and P. A. Brennan, "Accurate metallization capacitances for integrated circuits and packages," IEEE J. Solid-State Circuits, vol. SC-8, pp. 289-290, Aug. 1973.

[10] A. E. Ruehli andx P. A. Brennan, "Capacitance models for integrated circuit metallization wires," IEEE J. Solid-State Circuits, vol. SC-10, Dec, 1975.

[11] R. L. M. Dang and N. Shigyo, "Coupling capacitances for twodimensional wires," IEEE Electron Device Lett., vol. EDL-2, pp. 196-197, Aug. 1981.

[12] W. H. Chang, "Analytical IC metal-line capacitance formulas," IEEE Trans. Microwave Theory Tech., vol. MTT-24, pp. 608611 , Sept. 1976

[13] D. B. Scott, W. R. Hunter, and H. Shichijo, "A transmission line model for silicided diffusions: Impact on the performance of VLSI circuits," IEEE Trans. Electron Devices, vol. ED-29, pp. 651-661, Apr. 1982.

[14] A. K. Sinha, J. A. Cooper, and H. J. Levinstein, "Speed limitations due to interconnect time constants in VLSI integrated circuits," IEEE Electron Device Lett., vol. EDL-3, pp. 90-92, Apr. 1982.

[15] A. Mayadas and M. Sahtzkes, "Electrical resistivity model for polycrystalline films: The case of arbitrary reflection at ex ternal surface," Phys. Rev., B-1, pp. 1382-1389, 1970.

[16] A. Wilnai, "Open-ended RC line model predicts MOSFET IC response," EDN, pp. 53-54, Dec. 1971.

[17] T. Sakurai, "Approximation of wiring delay in MOSFET LSI," IEEE J. Solid-State Circuits, vol. SC-18, pp. 418-426, Aug. 1983.

[18] H. B. Bakoglu and J. D. Meindl, "Optimal interconnect circuits for VLSI," in ISSCC Dig. Tech. Papers, pp. 164-165, Feb. 1984.

[19] R. W. Keyes, "The wire-limited logic chip," IEEE J. Solid-State Circuits, vol. SC-17, pp. 1232-1233, Dec. 1982.

[20] C. Mead and M. Rem, "Minimum propagation delays in VLSI," IEEE J. Solid-State Circuits, vol. SC-17, pp. 773-775, Aug. 1982

[21] P. I. Suciu and R. L. Johnson, "Experimental derivation of the source and drain resistance of MOS transistors," IEEE Trans. Electron Devices, vol, ED-27, pp. 1846-1848, Sept. 1980.

[22] C. Y. Chang, Y. K. Fang, and S. M. Sze, "Specific contact resistance of metal-semiconductor barriers," Solid-State Electron., vol. 14, p. 541, 1971.

[23] D. Pramanik and A. N. Saxena, "VLSI metallization using aluminum and its alloys: Part I," Solid State Technol.,pp. 127133, Jan. 1983.

[24] - "VLSI metallization using aluminum and its alloys: Part II," Solid State Technol., pp. 131-138, Mar. 1983.

[25] C. L. Seitz, "Self-timed VLSI systems," in Proc. Caltech Conf. VLSI, C. L. Seitz, Ed., pp. 345-355, Jan. 1980.

[26] C. Mead and L. Conway, Introduction to VLSI Systems. Reading, MA: Addison-Wesley, $1980, \mathrm{ch} .7$.

[27] R. W. Keyes, "The evolution of digital electronics towards VLSI," IEEE Trans. Electron Devices, vol. ED-26, pp. 271-278, 1979. 